\section{Quantitative Investigation of C-F... $\pi$ Interaction in 4-(2-(((6-(trifluoromethyl) pyridin-2-yl)oxy)methyl)phenyl)-1,2-dihydro- 3H-pyrazol-3-one}

\section{Abstract}

In this study, we have investigated the nature and characteristics of C-F $\cdots \pi$ interactions present in the crystal structure of 4-(2-(((6-(trifluoromethyl)pyridin-2yl)oxy)methyl)phenyl)-1,2-dihydro-3H-pyrazol-3-one (1). This is a case of an $I p \cdots \pi$ interaction where the $I p$ of the fluorine interacts with the pyridinyl $\pi$-ring. The molecular pairs consisting of $\mathrm{C}-\mathrm{F} \cdot \cdots \pi$ interactions were stabilized with dispersion being the dominant force. The existence of these F $\cdots \pi$ interactions were further confirmed by the presence of $(3,-1)$ bond critical points between fluorine and the carbon atoms of the $\pi$-rings.

Keywords: Molecular; Crystal structure; Carbon atoms

Received: July 03, 2017; Accepted: August 07, 2017; Published: August 10, 2017

\section{Introduction}

Non-covalent interactions plays a very important role in molecular self-assembly and are very important aspect of supra-molecular chemistry [1,2]. In recent years, weak interactions involving organic fluorine have garnered significant attention due to its unique properties such as high electronegativity and small size $[3,4]$. It was previously believed that organic fluorine cannot participate in formation of hydrogen bond [5] but it has been now firmly established that fluorine can form highly directional and stabilized hydrogen bonds [6-8]. Apart from hydrogen bonds, fluorine can also participate in the formation of $F \cdot \cdots \pi$ interaction also where the $I p$ of the fluorine interact with $\pi$-rings [9]. F $\cdots \pi$ interactions has been observed to play an important role in molecular crystals and hence it is of interest to quantitatively investigate the nature and characteristics of $F \cdots \pi$ interactions $[7,10-12]$. In this study, we have quantitatively investigate the role of $\mathrm{C}-\mathrm{F} \cdot \cdots \pi$ interactions in the crystal structure of 4-(2-(((6-(trifluoromethyl)pyridin-2-yl) oxy)methyl)phenyl)-1,2-dihydro-3H-pyrazol-3-one (1). The aim of this study was to evaluate the strength of the $F \cdots \pi$ interaction and their contribution towards molecular packing. Topological analysis was also performed in order to evaluate the magnitude of the electron density $(\rho)$ and Laplacian $\left(\nabla^{2} \rho\right)$ at the bond critical point of the interacting atoms.

\section{Experimental Details}

The present compound [4-[2-(\{[6-(trifluoromethyl)pyridin2-yl]oxy\}methyl)phenyl]-1,2-dihydro-3H-pyrazol-3-one] was synthesized by the process of reductive cyclization, using $0.367 \mathrm{gm}$,

\section{Rahul Shukla', Chetan Shripanavar ${ }^{2}$ and Deepak Chopra ${ }^{1}$}

\section{Crystallography and Crystal Chemistry Laboratory, Department of Chemistry, Indian Institute of Science Education and Research, Bhopal, Madhya Pradesh, India \\ 2 Department of Agrochemicals and Pest Management, Devchand College, Arjunnagar, Karnataka, India}

\section{Corresponding author: \\ Deepak Chopra}

\section{झ dchopra@iiserb.ac.in}

Crystallography and Crystal Chemistry Laboratory, Department of Chemistry, Indian Institute of Science Education and Research, Bhopal, Madhya Pradesh, India.

Tel: +9107556691311

\section{Citation: Shukla R, Shripanavar C, Chopra D. Quantitative Investigation of C-F $\cdots \pi$ Interaction in 4-(2-(((6-(trifluoromethyl) pyridin-2-yl)oxy)methyl)phenyl)-1,2-dihydro- 3H-pyrazol-3-one. Struct Chem Crystallogr Commun. 2017, Vol 3 No.1:4}

0.001 mol of (2E)-3-methoxy-2-[2-(\{[6-(trifluoromethyl)pyridin-2$\mathrm{yl}$ loxy\}methyl)phenyl]prop-2-enehydrazide, which is dissolved in $10 \mathrm{ml}$ of methanol along with 2 drops of conc. $\mathrm{H}_{2} \mathrm{SO}_{4}$ (Scheme 1). The reaction mixture was refluxed for $6 \mathrm{~h}$ at $80^{\circ} \mathrm{C}$ temperature and cooled to separate out the final product. The final product is again re-crystallized by dissolving in ethanol and by the process of slow evaporation needle shaped white transparent crystals of the product were obtained. The cyclization of hydrazide is a wellknown process and has been utilized extensively for synthesizing a variety of pyrazol derivatives $[13,14]$.

\section{$\mathrm{X}$-ray data collection}

Single crystal X-ray measurements were carried out on a Bruker AXS Kappa Apex 2 CCD diffractometer using monochromated Mo-K $\alpha$ radiation $(\lambda=0.71073 \AA)$ in phi $(\phi)$ and omega $(\omega)$ scans 


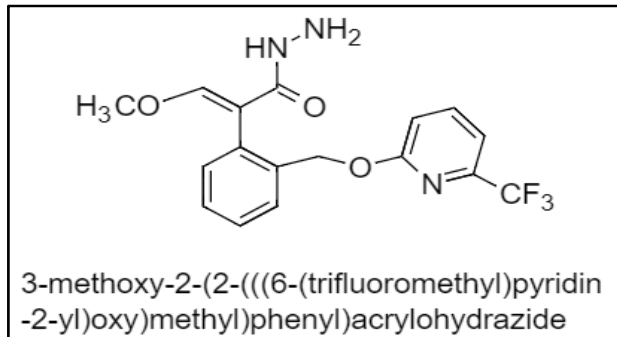

\section{Reductive Cyclization} $\mathrm{CH}_{3} \mathrm{OH}$, 2 drops Conc. $\mathrm{H}_{2} \mathrm{SO}_{4}$

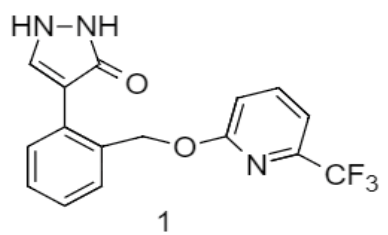

4-(2-(((6-(trifluoromethyl)pyridin-2-yl)oxy)methyl)phenyl) -1,2-dihydro-3H-pyrazol-3-one

Scheme 1 Schematic representation of synthesis of 4-[2-(\{[6-(trifluoromethyl)pyridin-2-yl] oxy $\}$ methyl)phenyl]-1,2-dihydro-3H-pyrazol3-one (1) with its chemical name.

at 100 (2) K. Unit cell measurement, data collection, integration, scaling and absorption corrections were performed using Bruker Apex II software [15]. The intensity data were processed by using the Bruker SAINT [16] suite of programs. The crystal structure was refined by the full matrix least squares method using SHELXL14 [17] present in the program suite WinGX [18]. Empirical absorption correction was applied using SADABS [19]. The $\mathrm{CF}_{3}$ group is disordered over two independent orientations and refines in the ratio of 2:1. The non-hydrogen atoms were refined anisotropically and the hydrogen atoms bonded to $\mathrm{C}$ and $\mathrm{N}$ atoms were positioned geometrically and refined using a riding model with $U_{i s o}(H)=1.2 U_{\text {eq }}(C, N)$. The molecular connectivity and the crystal packing diagrams were generated using the Mercury 3.9 (CCDC) program [20]. Geometrical calculations were done using PARST [21] and PLATON [22]. The details of data collection and crystal structure refinement are shown in Table 1.

\section{Theoretical calculations}

Lattice energy and intermolecular interaction energies partitioned into coulombic $\left(\mathrm{E}_{\text {coul }}\right)$, polarization $\left(\mathrm{E}_{\text {pol }}\right)$, dispersion $\left(\mathrm{E}_{\text {disp }}\right)$, and repulsion energy $\left(E_{r e p}\right)$ terms were evaluated using the PIXEL method present in the CLP module [23] which has been utilized in several similar studies. Crystal Explorer (version 17.5) software was also utilized for mapping 2D fingerprint plots [24] which helped in evaluating the contribution of different intermolecular interactions present in a molecule in the crystalline environment. Topological analysis was performed at B3LYP/6-311G** level of theory by using AIMALL software [25] which is based on Bader's Quantum Theory of Atoms in Molecules [26]. For the purpose of quantitative analysis, fluorine atoms with major occupancy were considered for our calculations.

\section{Results and Discussion}

Figure 1 shows the ORTEP of the title molecule which crystallizes in monoclinic $P Z_{1} / c$ space group with $Z=8$. One of the molecules present in the asymmetric unit consisted of a disordered $\mathrm{CF}_{3}$ group, with the occupancy of the major: minor orientation being in the ratio of $0.57: 0.43$. The lattice energy of 1 was calculated to be $-190.3 \mathrm{~kJ} / \mathrm{mol}$ with dispersion being the largest contributor towards the stabilization ( $42 \%$ ) followed by stabilization from coulombic ( $\sim 40 \%)$ and polarization ( $18 \%)$ contribution (Table 2). Analysis of the crystal structure reveals that there are five different molecular motifs which are involved the formation of $\mathrm{C}-\mathrm{F} \cdot \cdots \pi$ interactions (Figure 2; Table 3). The interaction energies
Table 1 Single crystal data collection and refinement details of 1.

\begin{tabular}{|c|c|}
\hline Empirical formula & $\mathrm{C}_{16} \mathrm{H}_{12} \mathrm{~F}_{3} \mathrm{~N}_{3} \mathrm{O}_{2}$ \\
\hline Formula weight & 335.29 \\
\hline Crystal system & Monoclinic \\
\hline Space group & $\mathrm{P} 21 / \mathrm{c}$ \\
\hline a $(\AA ̊)$ & $16.4436(17)$ \\
\hline$b(\AA)$ & $18.236(2)$ \\
\hline$c(\AA ̊)$ & $10.4744(11)$ \\
\hline$\alpha\left(^{\circ}\right)$ & 90 \\
\hline$\beta\left({ }^{\circ}\right)$ & $108.57(3)$ \\
\hline$\gamma\left({ }^{\circ}\right)$ & 90 \\
\hline$V(A 3)$ & 2977.3(5) \\
\hline $\mathrm{Z}, \mathrm{Z}^{\mathrm{c}}$ & 8,2 \\
\hline Dcalc $\left(\mathrm{gcm}^{-3}\right)$ & 1.496 \\
\hline$\mu\left(\mathrm{mm}^{-1}\right)$ & 0.126 \\
\hline$F(000)$ & 1376 \\
\hline$\theta \min , \theta \max$ & $2.588,27.626$ \\
\hline Treatment of Hydrogens & Fixed \\
\hline hmin, max, kmin, max, Imin, $\max$ & $-21,19,-23,23,-13,13$ \\
\hline No. of ref. & 24734 \\
\hline $\begin{array}{l}\text { No. of unique reflections/Observed } \\
\text { reflections }\end{array}$ & $6067 / 3278$ \\
\hline Goodness-of-fit & 1 \\
\hline Final R indices $[1>2 \sigma(I)]$ & $R 1=0.0965, w R 2=0.2434$ \\
\hline $\mathrm{R}$ indices (all data) & $R 1=0.1750, w R 2=0.2793$ \\
\hline $\begin{array}{l}\text { Largest difference peak and hole (e } \\
\qquad \AA-3)\end{array}$ & 0.55 and -0.52 \\
\hline CCDC reference number & 1559231 \\
\hline
\end{tabular}

Table $\mathbf{2}$ Lattice Energy of 1 partitioned into different energy components (in $\mathrm{kJ} / \mathrm{mol}$ ).

\begin{tabular}{|c|c|c|c|c|c|}
\hline Code & $\mathbf{E}_{\text {coul }}$ & $\mathbf{E}_{\text {pol }}$ & $\mathbf{E}_{\text {disp }}$ & $\mathbf{E}_{\text {rep }}$ & $\mathbf{E}_{\text {tot }}$ \\
\hline $\mathbf{1}$ & -154.9 & -72.2 & -163.5 & 200.3 & -190.3 \\
\hline
\end{tabular}

in these motifs ranged from $-25.1 \mathrm{~kJ} / \mathrm{mol}$ to $-3.7 \mathrm{~kJ} / \mathrm{mol}$ with dispersion being the largest contributor towards the stabilization (Table 3). The C-F $\cdots \pi(C)$ distance in these motifs were in the range of 2.93-3.77 $\AA$ while the $\mathrm{C}-\mathrm{F} \cdots \pi(\mathrm{C})$ directionality is in the range of $108-142^{\circ}$. It is important to note that in case of motif I, II and III that the total stabilization energy was significantly 


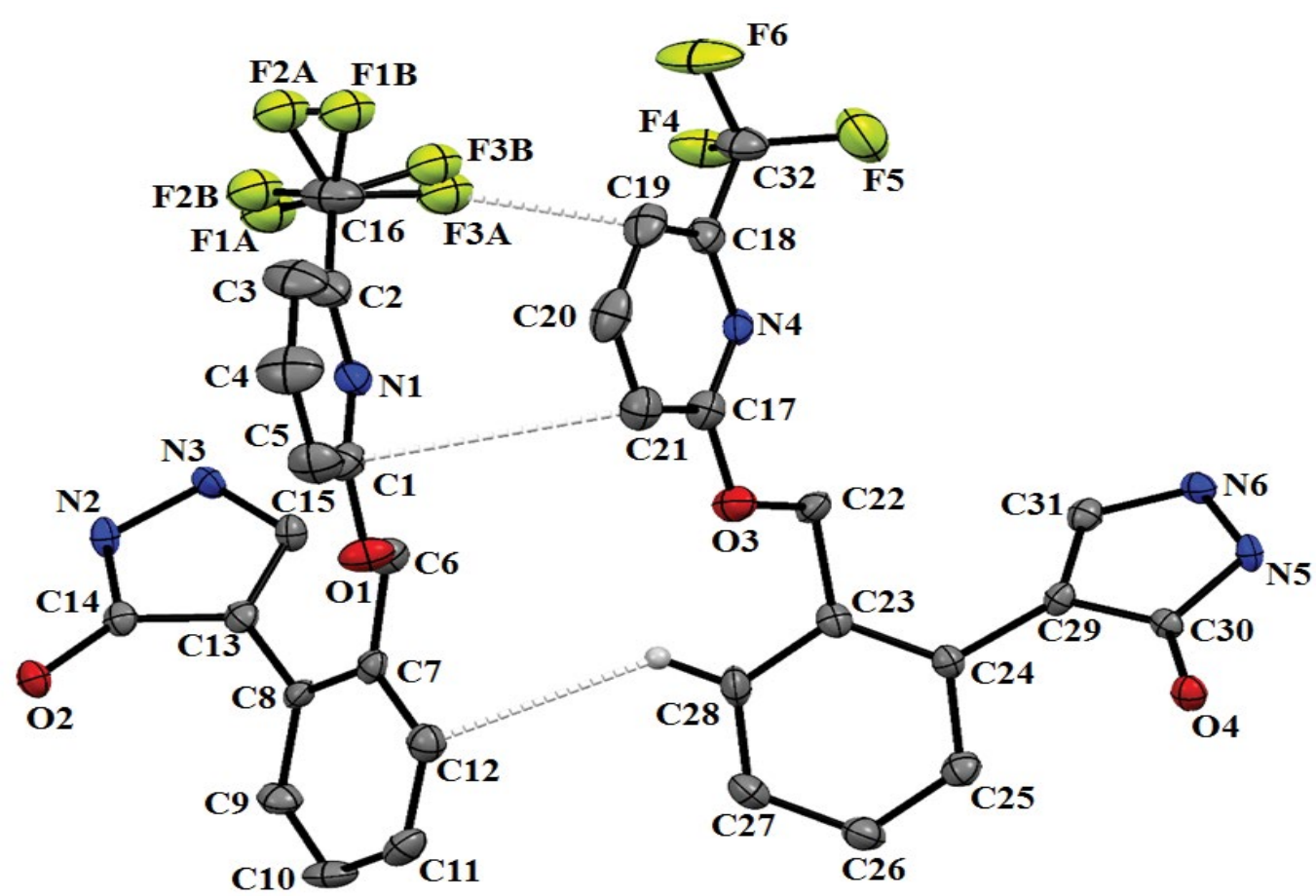

Figure 1 ORTEP of 1 drawn at 50\% ellipsoidal probability. Dotted lines indicate C...C, C-H... $\pi$ and C-F... $\pi$ contacts between two molecules in the asymmetric unit.

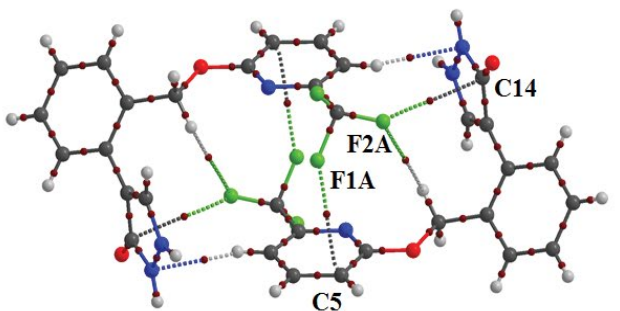

a) $\mathrm{I},-25.1 \mathrm{~kJ} / \mathrm{mol}$

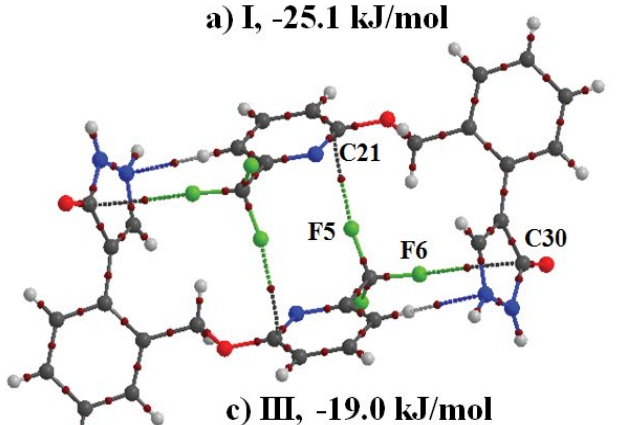

c) III, $-19.0 \mathrm{~kJ} / \mathrm{mol}$

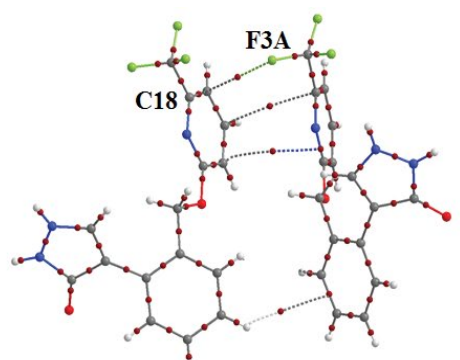

b) II, $-24.5 \mathrm{~kJ} / \mathrm{mol}$

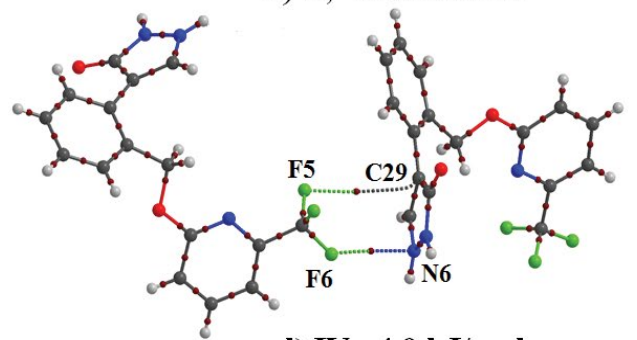

d) $\mathrm{IV},-4.9 \mathrm{~kJ} / \mathrm{mol}$

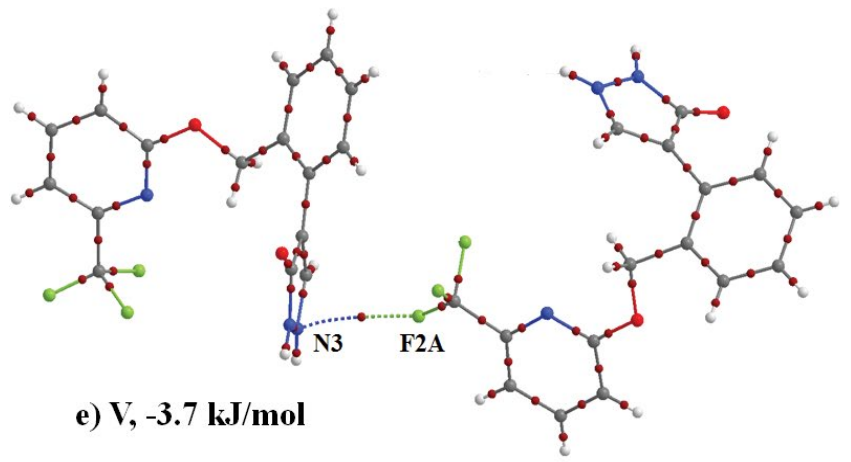

Figure 2 (a)-(e): Molecular graphs in 1 showing the existence of $F . . . C(\pi)$ bond critical points. 
larger as compared to the other two motifs due to the presence of hydrogen bonds in addition to the $F \cdot \cdots \pi$ interaction which provided additional stability to the respective motifs (Table 3 ).

The presence of the $F \cdots \pi(C)$ interactions in the above discussed motifs was further confirmed by a topological analysis due to the presence of a bond critical point between the interacting fluorine and the carbon atom of the $\pi$-ring (Figure 2). The F...C bond path length in these ranged from $2.937 \AA$ to $3.916 \AA$. It is important to note that while the magnitude of the bond path length was similar to that of bond length; their origin and physical significance were different. The magnitude of $\rho$ ranged from 0.013-0.055 e/ $\AA^{3}$ while the magnitude of $\nabla^{2} \rho$ ranged from $0.199-0.797$ e/ $\AA^{5}$ (Table 4). In general, the magnitude of both $\rho$ and $\nabla^{2} \rho$ decreases with increasing value of BPL. The low value of $\rho$ accompanied by positive value of Laplacian confirms $F \cdots C(\pi)$ interactions to be a case of closed-shell interactions. The Hirshfeld fingerprint plot also shows that $\mathrm{F} \cdots \mathrm{C}(\pi)$ interactions had $9.3 \%$ contribution towards the molecular packing and establish that $\mathrm{F} \cdot . \cdot \pi$ interaction indeed plays a very important role in the crystal packing of the title compound (Figure 3).

\section{Summary}

In this study, we have quantitatively investigated the role of $C-F \ldots \pi(C)$ interactions in the crystal structure of 4-(2-(((6-(trifluoromethyl)pyridin-2-yl)oxy)methyl)phenyl)-1,2dihydro-3H-pyrazol-3-one. It was evident that both dispersion and coulombic energy components had significant contribution towards the crystal lattice. Calculations on the interaction energy reveal that the motifs consisting of $C-F . . . \pi$ interactions were attractive in nature with dispersion being the dominant contributor towards stabilization. Topological calculations further confirmed the existence of these weak interactions. Future study will be focussed on analysing the interplay between $C-F . . . \pi$ and other non-covalent interactions, such as hydrogen bonds, present in the crystal structure of related molecules and their subsequent role in formation of the 3D molecular packing.

Table 3 List of molecular motifs consisting of C-F $\cdots \pi$ interactions along with interaction energies and geometrical parameters.

\begin{tabular}{|c|c|c|c|c|c|c|c|c|c|}
\hline Motif & $\begin{array}{l}\text { Centroid- } \\
\text { Centroid } \\
\text { Distance }\end{array}$ & Ecoul & Epol & Edisp & Erep & Etot & Symmetry & Interaction & $\begin{array}{l}\text { Geometrical } \\
\text { Parameters }\end{array}$ \\
\hline & & (kJ/mol) & (kJ/mol) & $(\mathrm{kJ} / \mathrm{mol})$ & (kJ/mol) & (kJ/mol) & & & $\left(\AA ̊ /{ }^{\circ}\right)$ \\
\hline \multirow[t]{3}{*}{1} & 5.869 & -1.1 & -11.8 & -52.3 & 40 & -25.1 & $2-x, 1-y,-z$ & C16-F1A...C5 & $3.237 / 141$ \\
\hline & & & & & & & & C16-F2A...C14 & $3.438 / 141$ \\
\hline & & & & & & & & C3-H3...N2 & $2.55 / 159$ \\
\hline \multirow[t]{3}{*}{ II } & 6.362 & -5.4 & -3.6 & -40.6 & 25.1 & -24.5 & $x, y, z$ & C16-F3A...C18 & $3.165 / 132$ \\
\hline & & & & & & & & $\mathrm{C} 28-\mathrm{H} 28 \ldots \mathrm{C} 12$ & $2.91 / 132$ \\
\hline & & & & & & & & $\pi(C 1) \ldots \pi(C 21)$ & 3.464 \\
\hline \multirow[t]{3}{*}{ III } & 5.732 & 6.7 & -8.5 & -48.6 & 31.4 & -19 & $1-x, 1-y,-z$ & C32-F6...C30 & $3.400 / 150$ \\
\hline & & & & & & & & C32-F5...C21 & $3.223 / 162$ \\
\hline & & & & & & & & C19-H19...N6 & $2.63 / 163$ \\
\hline \multirow[t]{2}{*}{ IV } & 10.444 & -0.2 & -1.2 & -9.4 & 5.9 & -4.9 & $\begin{array}{c}1-x,- \\
0.5+y, 0.5-z\end{array}$ & C32-F6...N6 & $2.932 / 137$ \\
\hline & & & & & & & & C32-F5...C29 & $3.777 / 107$ \\
\hline V & 10.313 & 2.4 & -1.2 & -10.5 & 5.6 & -3.7 & $\begin{array}{c}2-x,- \\
0.5+y, 0.5-z\end{array}$ & C16-F2A...N3 & $3.031 / 142$ \\
\hline
\end{tabular}
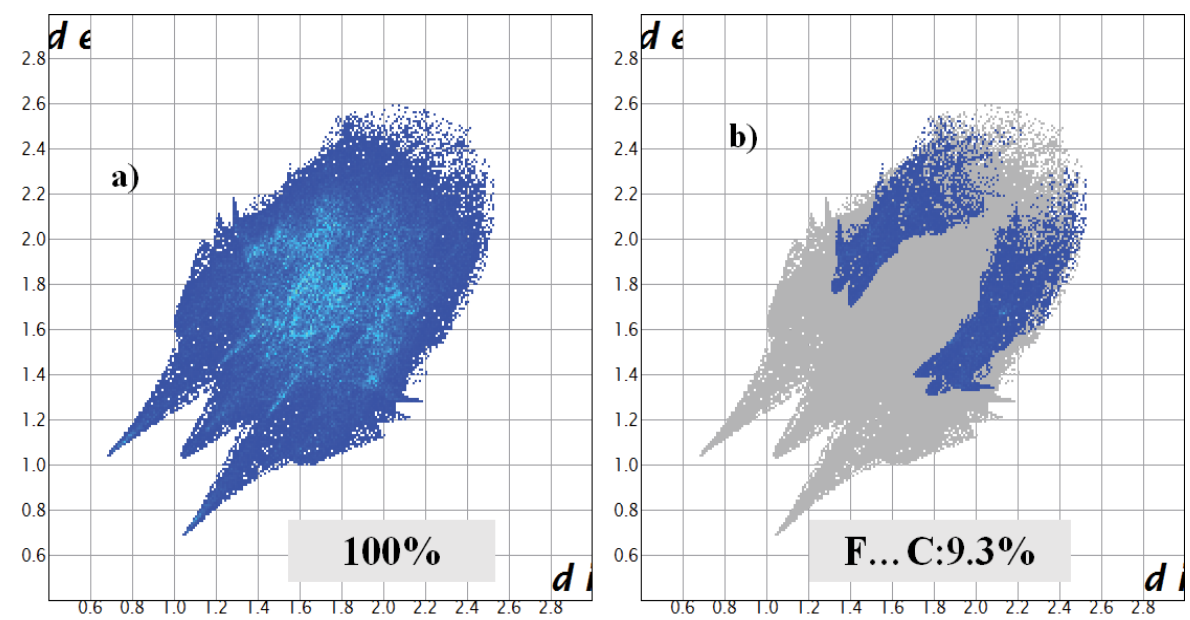

Figure 3 2D Fingerprint plot for 1 a) Overall b) F...C interaction. 
Table 4 Topological parameters obtained for different C-F... $\pi(C)$ interactions present in 1.

\begin{tabular}{|c|c|c|c|}
\hline Interaction & BPL (Å) & $\rho\left(\mathrm{e} / \AA^{3}\right)$ & $\nabla^{2} \rho\left(e / \AA^{5}\right)$ \\
\hline C32-F6...N6 & 2.937 & 0.055 & 0.797 \\
\hline C16-F2A...N3 & 3.056 & 0.046 & 0.697 \\
\hline C32-F5...C21 & 3.198 & 0.038 & 0.557 \\
\hline C16-F1A...C5 & 3.266 & 0.036 & 0.518 \\
\hline C32-F6...C30 & 3.409 & 0.018 & 0.301 \\
\hline C16-F2A...C14 & 3.445 & 0.017 & 0.290 \\
\hline C16-F3A...C18 & 3.784 & 0.043 & 0.555 \\
\hline C32-F5...C29 & 3.916 & 0.013 & 0.199 \\
\hline
\end{tabular}

\section{Acknowledgements}

RS thanks DST for INSPIRE-PhD Fellowship. DC thanks IISER Bhopal for infrastructural and research facilities. 


\section{References}

1 Bombicz P, Gruber T, Fischer C, Weber E, Kálmán A (2014) Fine tuning of crystal architecture by intermolecular interactions: synthon engineering. CrystEngComm 16: 3646-3654.

2 Desiraju GR (2000) Hydrogen bonds and other intermolecular interactions in organometallic crystals. J Chem Soc Dalton Trans: 3745-3751.

3 Chopra D, Guru Row TN (2011) Role of organic fluorine in crystal engineering. CrystEngComm 13: 2175-2186.

4 Chopra D (2012) Is Organic Fluorine Really "Not" Polarizable? Cryst Growth Des 12: 541-546.

5 Dunitz JD, Taylor R (1997) Organic Fluorine Hardly Ever Accepts Hydrogen Bonds. Chem Eur J 3: 89-98.

6 Thakur TS, Kirchner MT, Bläser D, Boese R, Desiraju GR (2010) C$\mathrm{H} \cdots \mathrm{F}-\mathrm{C}$ hydrogen bonding in 1,2,3,5-tetrafluorobenzene and other fluoroaromatic compounds and the crystal structure of alloxan revisited. CrystEngComm 12: 2079-2085.

7 Shukla R, Chopra D (2015) Crystallographic and computational investigation of intermolecular interactions involving organic fluorine with relevance to the hybridization of the carbon atom. CrystEngComm 17: 3596-6309.

8 Holl MG, Struble MD, Singal P, Seigler MA, Lectka T (2016) Positioning a Carbon-Fluorine Bond over the $\pi$ Cloud of an Aromatic Ring: A Different Type of Arene Activation. Angew Chem Int Ed 55: 82668269.

9 Panini P, Chopra D (2012) Role of intermolecular interactions involving organic fluorine in trifluoromethylatedbenzanilides. CrystEngComm 14: 1972-1989.

10 Lu YX, Zou JW, Wang YH, Yu QS (2007) Substituent Effects on Noncovalent Halogen/ $\pi$ Interactions: Theoretical Study. Int J Quant Chem 107: 1479-1486.

11 Li P, Maier JM, Vik EC, Yehl CJ, Dial BE, et al. (2017) Stabilizing Fluorine $-\pi$ Interactions. Angew Chem Int Ed 56: 1-5.

12 Scerba MT, Bloom S, Haselton N, Siegler M, Jaffe J, et al. (2012)
Interaction of a $\mathrm{C}-\mathrm{F}$ Bond with the $\pi$-System of a $\mathrm{C}=\mathrm{C}$ Bond or "Head On" with a Proximate C-H Bond. J Org Chem 77: 1605-1609.

13 Burbuliene MM, Bobrovas O, Vainilavicius $P$ (2006) Synthesis and intramolecular cyclization of 2-methylsulfany-4-oxo-3(4H)quinazolinyl)acetohydrazide. J Heterocyclic Chem 43: 43-47.

14 Majumdar P, Pati A, Patra M, Behera RK, Behera AK (2014) Acid Hydrazides, Potent Reagents for Synthesis of Oxygen-, Nitrogen-, and/or Sulfur-Containing Heterocyclic Rings. Chem Rev 114: 2942-2977.

15 Bruker Analytical X-ray Systems (2006) Apex2, Version 2 User Manual, M86-E01078, Madison, WI.

16 Siemens Analytical X-ray Instruments Inc (1995) Siemens, SMART System, Madison, MI.

17 Sheldrick G M (2008) A short of SHELX. Acta Crystallogr Sect. A: Found Crystallogr 64: 112-122.

18 Farrugia LJ (1999) WinGX suite for small-molecule single-crystal crystallography. J Appl Crystallogr 32: 837-838.

19 Sheldrick GM (2007) SADABS, Bruker AXS, Inc. Madison, WI.

20 Macrae CF, Bruno IJ, Chisholm JA, Edgington PR, McCabe P, et al. (2008) Mercury CSD 2.0 - New Features for the Visualization and Investigation of Crystal Structures. J Appl Cryst 41: 466-470.

21 Nardelli M (1995) PARST95-an update to PARST: a system of Fortran routines for calculating molecular structure parameters from the results of crystal structure analyses. J ApplCryst 28: 659-659.

22 Spek AL (2009) Structure validation in chemical crystallography. ActaCrystD 65: 148-155.

23 Dunitz JD, Gavezzotti A (2012) Supramolecular synthons: validation and ranking of intermolecular interaction energies. Cryst Growth Des 12: 5873-5877.

24 Turner MJ, McKinnon JJ, Wolff SK, Grimwood DJ, Spackman PR, et al. (2017) Crystal Explorer 17.

25 Keith TA (2013) AIMALL, version 13.05.06, TK Gristmill Software, Overland Park, KS, USA.

26 Bader R (1990) Atoms in Molecules: A Quantum Theory, International Series of Monographs on Chemistry. Clarendon Press. 